\title{
Introduction du numéro thématique « Economie de la connaissance sur le Rhin supérieur »
}

\section{Rita Schneider-Sliwa}

\section{(2) OpenEdition}

\section{Journals}

Édition électronique

URL : http://journals.openedition.org/rge/1682

DOI : $10.4000 /$ rge. 1682

ISSN : 2108-6478

Éditeur

Association des géographes de l'Est

Édition imprimée

Date de publication : 1 septembre 2008

ISSN : 0035-3213

Référence électronique

Rita Schneider-Sliwa, « Introduction du numéro thématique « Economie de la connaissance sur le Rhin supérieur » », Revue Géographique de l'Est [En ligne], vol. 48 / 3-4 | 2008, mis en ligne le 02 mars 2010, consulté le 08 septembre 2020. URL : http://journals.openedition.org/rge/1682 ; DOI : https://doi.org/ $10.4000 /$ rge. 1682

Ce document a été généré automatiquement le 8 septembre 2020.

Tous droits réservés 


\title{
Introduction du numéro thématique «Economie de la connaissance sur le Rhin supérieur »
}

\author{
Rita Schneider-Sliwa
}

1 La connaissance constitue « la ressource du XXIème siècle ». Elle est « vitale pour notre avenir ». Ainsi s'exprimaient récemment D.B. Audretsch et E.E. Lehmann, deux spécialistes de l'innovation de l'Institut Max-Planck d'économie dans leur article intitulé «Les universités et la croissance économique régionale » (Rapport d'activité 2005. Max-Planck-Gesellschaft. Max-Planck-Institut für Ökonomik, Iéna, 2005). L'innovation est un ferment de la compétitivité mondiale, de la stabilité de la croissance économique et un garant de haute qualité de vie. Une forte insertion dans le monde du travail, une productivité supérieure à la moyenne ainsi qu'un investissement dans la recherche et le développement sont également des éléments clés, ainsi qu'une bonne connexion entre la connaissance et la production. Le devenir des pays ou des régions ainsi que l'aménagement d'espaces économiques prospères et ouverts sur l'international passe par un renforcement de la recherche et de la formation, qu'il s'agisse d'activités scientifiques, industrielles ou financières. Les universités, l'Etat, l'économie et la société doivent entreprendre des efforts communs en ce sens.

2 Cette réflexion n'est certes pas nouvelle. Elle repose sur une approche standard de la recherche empirique établie en 1957, selon laquelle la croissance économique repose sur le progrès technique, considéré comme la somme de toutes les améliorations technologiques et organisationnelles, et produit par le biais de l'innovation au sens large (Solow 1956, 1957). Il apparaît en particulier qu'une grande partie des différentiels de croissance et de revenus observés entre les économies nationales et régionales peut être expliquée en considérant le capital humain et la qualification de la main-d'œuvre comme les moteurs du progrès technique. Ce constat pionnier a été suffisamment retentissant pour assurer à son auteur, Robert Merton Solow, le prix Nobel d'économie en 1987 pour son travail sur la théorie de la croissance économique élaboré entre 1956 et 1957. 
3 Ce qui est nouveau, en revanche, c'est que la société, la politique et le grand public ont reconnu, depuis les années 1990, que la croissance économique ne résultait plus de la production de masse effectuée par de grandes entreprises comme durant l'ère industrielle, mais que les sources de la compétitivité, de la création d'emplois et du bien-être s'étaient transformées. Il est aujourd'hui reconnu que les technologies de l'information et de la communication ont contribué à modifier la concurrence internationale. Deux des piliers de l'économie entrepreneuriale (entrepreneurship economy) sont internationalement reconnus comme les moteurs de la croissance économique : l'innovation par l'investissement dans la R\&D et l'esprit d'entreprise, dont les processus conduisant à l'utilisation de la connaissance ont été fortement transformés par l'innovation.

4 Des recherches empiriques conduites récemment montrent que les régions qui investissent le plus dans la connaissance sont également celles qui possèdent la capacité d'innovation la plus forte (Audretsch 2005). Ces travaux suggèrent que la capacité d'innovation ne repose pas uniquement dans les entreprises mais doit aussi être produite par d'autres acteurs, comme les universités et les hautes écoles spécialisées qui produisent des effets d'entraînements de la connaissance (knowledge spillovers). Ils montrent également que les efforts politiques et sociaux doivent concourir à promouvoir une "économie de la connaissance» et une capacité d'innovation régionale, décisives pour la croissance économique. La recherche et le développement conduisent à la richesse et à la prospérité selon des principes bien établis et non pas par hasard, ce que montre l'essor contemporain de la Chine et la croissance économique de l'Inde. La promotion de la recherche et du développement devrait constituer les thèmes prioritaires de toutes les administrations publiques. Si rien n'est fait pour encourager ces activités, de graves conséquences sont à prévoir dans le domaine économique et social, car la promotion de la connaissance est synonyme d'un accroissement qualitatif et quantitatif du travail, d'une amélioration de l'égalité des chances et de la compétitivité. L'investissement public dans la formation et la recherche ainsi que l'existence de conditions cadres stables et créatives sont indispensables pour promouvoir la valeur économique du travail qualifié et de l'innovation et assurer le maintien de la compétitivité et d'un haut niveau de vie. Pour atteindre cet objectif, les universités, l'Etat, l'économie et la société doivent s'unir pour emprunter une voie difficile.

5 Ce numéro traite de certains aspects de l'économie de la connaissance dans le sud du Rhin supérieur. Cette région est depuis longtemps reconnue comme un foyer d'innovation et de connaissance d'envergure internationale, comme le rappellent les exemples historiques de l'Humanisme ou de l'Imprimerie au XVème siècle. Plus récemment, le Rhin supérieur a su jouer de sa situation centrale dans l'espace européen alors même qu'il était considéré comme une région périphérique des espaces nationaux de Suisse, de France ou d'Allemagne. Depuis les années 1990, par exemple, cette région s'est favorablement positionnée en matière d'avantages comparatifs dans le domaine des sciences de la vie, par le biais de l'initiative BioValley soutenue par les administrations publiques.

6 Ce numéro thématique se consacre aux tentatives destinées à impulser une dynamique à l'économie de la connaissance du Rhin supérieur. Dans la contribution de G. Wackermann intitulée "Démarches transfrontalières pionnières sur le Rhin Supérieur. Une rétrospective des débuts du façonnement d'un paysage européen du savoir » l'auteur montre 
quelles sont les étapes qui ont été franchies durant les cinquante dernières années pour faire du sud du Rhin supérieur une région compétitive au niveau européen. Il apparaît que ces dernières décennies ont permis de mettre en place un processus qui a conduit à bâtir une région européenne sur des bases solides et grâce à l'inlassable travail d'un grand nombre d'acteurs, mais qui a encore des faiblesse à surmonter.

7 La contribution de C. Klöpper et T. Haisch intitulée "Evolution de l'industrie biotech et medtech suisse et influence de l'industrie pharmaceutique sur le système d'innovation" examine l'influence de l'industrie pharmaceutique et des entreprises situées en amont et en aval sur le développement régional. Cette influence dépend de la façon dont ces industries sont insérées dans le contexte institutionnel national et de la manière dont elles peuvent développer leurs activités de recherche et de développement au plan régional.

8 La contribution de C. Klöpper intitulée «Structure et géographie des réseaux d'innovation. Une analyse des processus d'innovation des entreprises de biotechnologies de Bâle et de Strasbourg " traite des processus d'innovation au travers d'une approche bibliométrique comparée au plan régional. Les résultats montrent que les deux sous-parties du Rhin supérieur diffèrent en termes d'économie de la connaissance, ce qui permet de mettre en évidence une dépendance au sentier (path-dependency) liée aux contextes institutionnels et aux priorités politiques. Un constat similaire anime la contribution de R. Schneider-Sliwa intitulée «Enjeux et transformations des régions métropolitaines du sud $d u$ Rhin supérieur». Dans cette contribution, l'auteur pose la question de savoir si les politiques urbaines pourraient jouer un rôle décisif dans l'élaboration de nouveaux modèles de développement des régions urbaines, à la fois tournées vers l'avenir et compétitives. La contribution met en évidence plusieurs approches institutionnelles contemporaines destinées à favoriser le développement des régions métropolitaines dans les agglomérations de Strasbourg et de Bâle. L'agglomération de StrasbourgOrtenau est considérée ici comme un modèle qui permet de montrer quelles sont les structures institutionnelles et les compétences contraignantes qui doivent être institutionnalisées pour donner naissance à une région métropolitaine transfrontalière fonctionnelle. A Bâle, en revanche, le modèle de développement métropolitain s'appuie sur de faibles contraintes et sur des responsabilités qui ne sont pas partagées. En conséquence, les contributions suivantes se consacrent aux différents essais qui ont eu lieu pour renforcer la compétitivité de la région bâloise.

9 La contribution intitulée «Les jumelages stratégiques au service de la compétition entre les villes » de K. Bucher et N. Grillon examine la manière dont le canton de Bâle-Ville essaie de faire face aux nouveaux défis qui se posent aux régions urbaines au moyen de modèles d'action alternatifs et de formes organisationnelles adaptées. Ainsi, Bâle a mis en place, dans le cadre de sa réforme de l'administration publique, un office de la Ville (Stadtpräsidialamt) qui s'occupe de la stratégie de la politique extérieure de Bâle. La contribution montre quels sont les champs d'action actuels du canton en matière de politique extérieure dans le contexte de l'Agglomération Trinationale de Bâle. En s'appuyant sur le cas du jumelage entre Bâle et le Massachusetts, elle détaille ensuite quel rôle peuvent jouer les coopérations dans la stratégie de développement de la ville intitulée Basel 2020.

10 La contribution de B. Wieland intitulée "Fuite ou accueil des cerveaux? Une analyse de l'évolution du lieu de résidence des diplômés de l'Université de Bâle (2005) » étudie le parcours professionnels de jeunes diplômés et la manière dont la région bâloise se construit 
autour de l'économie de la connaissance. En calculant la proportion et la composition des universitaires qui, cinq ans après l'obtention de leur diplôme, habitent toujours ou sont revenus vivre dans la région de Bâle, l'auteur étudie l'attractivité du marché du travail régional pour les personnes hautement qualifiées. Sur la base de données provenant de l'office fédéral de la statistique suisse, il montre que les diplômés de l'Université de Bâle sont encore plus de $60 \%$ à habiter à proximité du lieu où ils ont fait leurs études, ce qui démontre l'attractivité de l'université en question et témoigne de la force du marché du travail régional pour les individus hautement qualifiés.

11 L'article de C. Saalfrank intitulé "L'économie et le milieu scientifique bâlois vus par les entreprises de haute technologie et les laboratoires de recherche " rappelle quant à lui que Bâle-Ville comptait 9521 entreprises et 178657 emplois salariés en 2003. L'analyse porte sur les entreprises et les laboratoires de recherche à forte intensité de connaissance qui font figure de locomotives pour le développement économique régional et national. Une enquête permet de récolter des données sur l'image de Bâle-Ville du point de vue de la localisation des activités scientifiques et économiques et d'en déduire des recommandations concrètes destinées à la promotion économique et au marketing urbain.

Dans l'ensemble, les contributions de ce numéro thématique présentent une image différenciée et hétérogène de l'économie de la connaissance et de sa capacité à promouvoir le développement économique régional. Il apparaît que les théories relatives au développement économique n'ont de portée que si les acteurs régionaux sont prêts à fixer des priorités et à agir eux-mêmes de manière innovante.

\section{AUTEUR}

\section{RITA SCHNEIDER-SLIWA}

Institute of Geography - Human Geography / Urban and Regional Studies - Department of Environmental Sciences, University of Basel - Klingelbergstrasse 27, CH 4056 Basel -

Rita.Schneider-Sliwa@unibas.ch 